\title{
Absorption properties of identical atoms
}

\author{
Pedro Sancho \\ Centro de Láseres Pulsados, CLPU \\ E-37008, Salamanca, Spain
}

\begin{abstract}
Emission rates and other optical properties of multiparticle systems in collective and entangled states differ from those in product ones. We show the existence of similar effects in the absorption probabilities for (anti)symmetrized states of two identical atoms. The effects strongly depend on the overlapping between the atoms and differ for bosons and fermions. We propose a viable experimental verification of these ideas.
\end{abstract}

Keywords: Optical properties of atomic (anti)symmetrized states; Identical atoms; Absorption rates

\section{Introduction}

The emission properties of multi-particle systems depend on the quantum state of the system. The first example of this behavior was presented by Dicke in his work on superradiance $[1,2,3]$ : a system in a collective state can radiate faster. The presence of collective states does not only change the emission properties, but also the absorption ones [4]. Later, it has been both theoretically and experimentally established the existence of modifications of the emission properties in the case of initially entangled states $[2,5,6,7]$. Other optical properties of atoms, such as Raman scattering, can also depend on the entangled character of the initial state [8]. In a related context, it has been demonstrated that the two-photon absorption rate can be modified when the photons are entangled [9].

In this work we show that another type of multi-particle state, the (anti) symmetrized state of identical particles, also leads to modifications of the absorption properties. In particular, we derive the absorption probabilities for systems of two identical particles, which in some cases differ from those corresponding to product states. These differences are essentially determined by the overlapping between the two atoms. Moreover, these modifications of the absorption probabilities are not similar for bosons and fermions. 
We propose a scheme for the experimental verification of the effect discussed in this work. A qualitative verification (involving a large number of atoms instead of only two) can, in principle, be obtained with minor modifications of existent techniques in the fields of atom lasers and (anti)bunching verification. The two-particle case could be experimentally studied with the same type of technique, but it is more demanding.

\section{Two-particle states}

In this section we determine the initial and final states of the atoms involved in the absorption process. We shall restrict our considerations to two-atom systems. The two atoms interact with light, for instance, a laser beam. We assume the intensity of the laser not to be too high. This way, only one-photon absorptions are relevant and we can neglect multi-photon processes.

The state of the atoms can be expressed as $\left|\psi_{g}\right\rangle=|\psi\rangle \mid g>$ or $\left|\psi_{e}\right\rangle=$ $|\psi>| e>$, with $\mid \psi>$ denoting the center of mass (CM) state, and $|g\rangle$ and $\mid e>$ representing the ground and excited states of the internal variables of the atom. We assume the laser to be tuned to the frequency associated with that transition. With this notation the initial state of the two-particle system is

$$
\begin{array}{r}
\mid \Psi_{i}>=N_{i}\left(\left|\phi_{g}>_{1}\right| \psi_{g}>_{2} \pm\left|\psi_{g}>_{1}\right| \phi_{g}>_{2}\right)= \\
N_{i}\left(\left|\phi>_{1}\right| \psi>_{2} \pm\left|\psi>_{1}\right| \phi>_{2}\right)\left|g>_{1}\right| g>_{2}
\end{array}
$$

with the upper and lower signs in the double sign expression corresponding respectively to bosons and fermions. The normalization factor is given by

$$
N_{i}=\frac{1}{\left(2\left(1 \pm|<\phi| \psi>\left.\right|^{2}\right)\right)^{1 / 2}}
$$

Let us now derive the final state after the absorption process. We consider first the case where the particle in state $\mid \phi_{g}>$ absorbs one photon. There are two changes in the atom. On the one hand, the internal state $\mid g>$ goes to the excited one $\mid e>$. On the other hand, the state of the center of mass also changes as $|\phi>\rightarrow| \tilde{\phi}>$. This process is a consequence of the recoil of the atom. Similarly, for the other state we have the evolution $\left|\psi_{g}>\rightarrow\right| \tilde{\psi}_{e}>$ after the photon absorption. Thus, the final state must contain $\tilde{\phi}_{e}$ and $\tilde{\psi}_{e}$ as the excited states, and $\phi_{g}$ and $\psi_{g}$ as the non-excited ones.

When the two final CM states are different, $\tilde{\phi}_{e}$ and $\tilde{\psi}_{e}$ are distinguishable alternatives to describe the two-particle system with one of the atoms in an excited state. After the absorption the system is not in a superposition of the two alternatives but in a mixture of the states representing them: $\mid \Psi_{f}(\tilde{\phi})>$ and $\mid \Psi_{f}(\tilde{\psi})>$, where the symbol between parentheses indicates the final CM state of the excited atom. Their explicit expressions are

$$
\mid \Psi_{f}(\tilde{\phi})>=\frac{1}{\sqrt{2}}\left(\left|\tilde{\phi}_{e}>_{1}\right| \psi_{g}>_{2} \pm\left|\psi_{g}>_{1}\right| \tilde{\phi}_{e}>_{2}\right)
$$


and

$$
\mid \Psi_{f}(\tilde{\psi})>=\frac{1}{\sqrt{2}}\left(\left|\tilde{\psi}_{e}>_{1}\right| \phi_{g}>_{2} \pm\left|\phi_{g}>_{1}\right| \tilde{\psi}_{e}>_{2}\right)
$$

There is a particular case that must be considered separately, that when the final states are equal, $|\tilde{\psi}>=| \tilde{\phi}>$ (because of the Pauli exclusion principle this case only refers to bosons). This situation clearly also corresponds to equal initial states, $|\psi\rangle=|\phi\rangle$. We cannot know if the absorption process has taken place via the particle labeled as 1 or that as 2 . These two alternatives are indistinguishable and the final state of the system must be a superposition of the states representing them:

$$
\mid \Psi_{f}(e q)>=\frac{1}{\sqrt{2}}\left(\left|\tilde{\phi}_{e}>_{1}\right| \phi_{g}>_{2}+\left|\phi_{g}>_{1}\right| \tilde{\phi}_{e}>_{2}\right)
$$

This form automatically fulfills the symmetrization condition. The fundamental difference with the case of non equal CM states is that now we have a pure state describing the two-particle system whereas previously we had a mixture.

\section{Absorption probabilities}

After deriving the initial and final states of the two-atom system we can easily obtain the matrix elements and probabilities for the absorption. The transition matrix elements for this process can be evaluated as

$$
\mathcal{M}_{\mathcal{F}}=<\Psi_{\mathcal{F}}\left|\hat{U}_{12}\right| \Psi_{i}>
$$

where $\left|\Psi_{\mathcal{F}}\right\rangle$ denotes any of the possible final states and $\hat{U}_{12}$ is the evolution operator of the two-particle system. Note that for the matter of simplicity we have not included in the above expression the variables associated with the electromagnetic field. In the initial state we should add $\mid n>_{E M}$, and in the final one $\mid n-1>_{E M}$. In the usual dipole and rotating-wave approximations [10], and to first order of perturbation theory the complete matrix element would read proportional to $\left.<\Psi_{\mathcal{F}}\left|\hat{U}_{12}\left(\hat{\mathbf{D}}_{1}, \hat{\mathbf{D}}_{2}\right)\right| \Psi_{i}><n-1|\hat{\mathbf{E}}| n\right\rangle_{E M}$ with $\hat{\mathbf{E}}$ the electric field operator, and $\hat{\mathbf{D}}_{1}$ and $\hat{\mathbf{D}}_{2}$ the dipole operators.

The evolution operator can be expressed as

$$
\hat{U}_{12}=\hat{U}_{1} \otimes \hat{U}_{2}
$$

with $\hat{U}_{i}, i=1,2$, the evolution operators of the particles. In order to simplify the problem and to capture the main physical ideas we neglect the atom-atom interactions. Then we can take $\hat{U}_{i}$ as the evolution operator of the particle only in interaction with the radiation field. Moreover, as the two particles are identical the two evolution operators are equal $\hat{U}_{1}=\hat{U}_{2}=\hat{U}$, and we can write $\hat{U}_{12}=\hat{U} \otimes \hat{U}$. 
Note that we do not explicitly include in $\hat{U}_{12}$ the initial and final times of the evolution $\left(\hat{U}_{12}\left(t_{f}, t_{i}\right)\right)$. We take $t_{f}-t_{i}$ as the duration of the laser pulse in each repetition of the experiment, which we assume to be short enough to avoid multiple absorption processes (such as absorption-spontaneous emissionabsorption or two absorptions processes) that would complicate the description of the system.

Let us compare the probabilities of having the particles in the final states $\tilde{\phi}_{e}$ and $\psi_{g}$ after the absorption process for two-atom systems in (anti)symmetrized and product states. The transition matrix element for the (anti)symmetrized state is $\mathcal{M}_{\tilde{\phi}}=<\Psi_{f}(\tilde{\phi})\left|\hat{U}_{12}\right| \Psi_{i}>$, which can be expressed as

$$
\mathcal{M}_{\tilde{\phi}}=\frac{<\tilde{\phi}_{e}|\hat{U}| \phi_{g}><\psi_{g}|\hat{U}| \psi_{g}> \pm<\tilde{\phi}_{e}|\hat{U}| \psi_{g}><\psi_{g}|\hat{U}| \phi_{g}>}{\left(1 \pm|<\phi| \psi>\left.\right|^{2}\right)^{1 / 2}}
$$

This probability amplitude contains two contributions, the direct one associated with the evolution

$$
\phi_{g} \rightarrow \tilde{\phi}_{e} ; \psi_{g} \rightarrow \psi_{g}
$$

and the crossed one representing the alternative evolution

$$
\psi_{g} \rightarrow \tilde{\phi}_{e} ; \phi_{g} \rightarrow \psi_{g}
$$

All the matrix elements in the numerator of Eq. (8) correspond to the singleparticle probability amplitudes for the evolutions in expressions (9) and (10). Then when the probability is evaluated, we have

$$
\begin{array}{r}
\left(1 \pm|<\phi| \psi>\left.\right|^{2}\right) P_{t w o}(\tilde{\phi})=P_{\text {sin }}\left(\phi_{g} \rightarrow \tilde{\phi}_{e}\right) P_{\text {sin }}\left(\psi_{g} \rightarrow \psi_{g}\right)+ \\
P_{\sin }\left(\psi_{g} \rightarrow \tilde{\phi}_{e}\right) P_{\sin }\left(\phi_{g} \rightarrow \psi_{g}\right) \pm \\
2 \operatorname{Re}\left(\mathcal{M}_{\text {sin }}^{*}\left(\phi_{g} \rightarrow \tilde{\phi}_{e}\right) \mathcal{M}_{\text {sin }}^{*}\left(\psi_{g} \rightarrow \psi_{g}\right) \mathcal{M}_{\sin }\left(\psi_{g} \rightarrow \tilde{\phi}_{e}\right) \mathcal{M}_{\sin }\left(\phi_{g} \rightarrow \psi_{g}\right)\right)
\end{array}
$$

where $P_{t w o}(\tilde{\phi})=\left|\mathcal{M}_{\tilde{\phi}}\right|^{2}$ is the probability of the two-particle system to be in the final state $\Psi_{f}(\tilde{\phi})$ when starting in $\Psi_{i}, P_{\text {sin }}\left(\phi_{g} \rightarrow \tilde{\phi}_{e}\right)$ represents the probability of a single atom evolving from $\phi_{g}$ to $\tilde{\phi}_{e}, \ldots$ The last term in the r. h. s. represents the interference effects between the two alternatives. This is a manifestation of the exchange effects.

There is a particular scenario where we can have a more clear picture. It corresponds to the case when the second term in the numerator of Eq. (8) can be neglected in comparison with the first one. This situation corresponds, for instance, to evolutions such that the evolved $\phi$ without absorption is very different from $\psi$.

In this scenario the matrix element simplifies to

$$
\mathcal{M}_{\tilde{\phi}} \approx \frac{<\tilde{\phi}_{e}|\hat{U}| \phi_{g}><\psi_{g}|\hat{U}| \psi_{g}>}{\left(1 \pm|<\phi| \psi>\left.\right|^{2}\right)^{1 / 2}}
$$


We want to compare this probability amplitude with that of a pair of identical atoms in factorized states. In this case the initial state is $\left|\Psi_{i}^{f a c}\right\rangle=\mid \psi_{g}>_{1}$ $\mid \phi_{g}>_{2}$, and the final one $\left|\Psi_{f}^{f a c}(\tilde{\phi})>=\right| \psi_{g}>_{1} \mid \tilde{\phi}_{e}>_{2}$. We have the transition probability amplitude $\mathcal{M}_{\tilde{\phi}}^{f a c}=<\Psi_{f}^{f a c}(\tilde{\phi})\left|\hat{U}_{12}\right| \Psi_{i}^{f a c}>$, which leads to the probability

$$
P_{t w o}^{f a c}(\tilde{\phi})=P_{s i n}\left(\phi_{g} \rightarrow \tilde{\phi}_{e}\right) P_{s i n}\left(\psi_{g} \rightarrow \psi_{g}\right)
$$

with $P_{t w o}^{f a c}(\tilde{\phi})$ denoting the probability of the two-particle system to be in the final state $\Psi_{f}^{f a c}(\tilde{\phi})$ when initially was in $\Psi_{i}^{f a c}$.

The ratio of the two probabilities is

$$
\frac{P_{t w o}(\tilde{\phi})}{P_{t w o}^{f a c}(\tilde{\phi})} \approx \frac{1}{1 \pm|<\phi| \psi>\left.\right|^{2}}
$$

The ratio strongly depends on the initial overlapping of the two atoms (such as measured by $|\langle\phi \mid \psi\rangle|^{2}$ ). We observe an opposite behaviour for bosons and fermions. In the first case, the absorption probability in the symmetrized state is smaller than in the product one. In contrast, for fermions, the ratio is larger than unity.

In the limit of negligible overlapping these effects tend to disappear, $P_{t w o}(\tilde{\phi}) \approx$ $P_{t w o}^{f a c}(\tilde{\phi})$.

We have only considered the problem of absorption of a photon with final states $\tilde{\phi}$ and $\psi$. The case of absorption with final states $\tilde{\psi}$ and $\phi$ is similar. Finally, the case in which the absorption can give rise to any of the pairs of final states, also easily follows taking into account that the two final states are distinguishable and the probabilities add.

When the second term in the numerator of Eq. (8) is comparable to the first one, one must deal with the complete equation. This situation occurs, for instance, for large overlappings. We shall only consider the limiting case of very similar initial $\mathrm{CM}$ wavefunctions, $\psi \approx \phi$. In this case we have $\mid<$ $\phi|\psi>|^{2} \approx 1$ and $\mathcal{M}_{\tilde{\phi}} \approx(1 \pm 1)<\tilde{\phi}_{e}|\hat{U}| \phi_{g}><\psi_{e}|\hat{U}| \psi_{g}>/(1 \pm 1)^{1 / 2}$. For fermions this expression is undefined, reflecting the Pauli principle. On the other hand, for bosons gives $P_{t w o}(e q)=2 P_{\sin }\left(\phi_{g} \rightarrow \tilde{\phi}_{e}\right) P_{s i n}\left(\phi_{g} \rightarrow \phi_{g}\right)$, expressing $P_{\text {two }}(e q)$ the probability of one absorption in the two-particle system when the two wavefunctions of the symmetrized initial state are equal. For a factorized two-particle state we have that the absorption can happen for $\mid \phi_{g}>_{1}$ and $\mid \phi_{g}>_{2}$. Then we have $P_{t w o}^{f a c}(e q)=2 P_{\text {sin }}\left(\phi_{g} \rightarrow \tilde{\phi}_{e}\right) P_{\text {sin }}\left(\phi_{g} \rightarrow \phi_{g}\right)$ and $P_{t w o}(e q)=P_{t w o}^{f a c}(e q)$, with $P_{t w o}^{f a c}(e q)$ the probability of one absorption in the twoparticle system when the two initial wave functions are equal and the initial and final states are factorized ones. In the limit of equal initial CM states there are not differences between symmetrized and factorized states. Note that the last result can also be derived starting directly from the state $\mid \Psi_{f}(e q)>$ introduced in Eq. (5), which gives $\mathcal{M}_{e q}=\sqrt{2}<\tilde{\phi}_{e}|\hat{U}| \phi_{g}><\phi_{g}|\hat{U}| \phi_{g}>$ 


\section{A proposal for an experimental test}

We discuss in this section if the effects considered above could be tested experimentally. We propose a scheme where it is possible, in principle, to carry out an experimental verification of the problem. Our scheme closely follows some ideas of the arrangements used in atom laser experiments and the verification of (anti)bunching effects (see, for instance, [11] for the first field and [12] for the second). We shall discuss separately two versions of the scheme. The first one only could provide a qualitative verification, but almost does not imply modifications of the part of the arrangement [11] that we use. The second one is much more demanding from the technical point of view, but could test the two-atom case.

\subsection{The qualitative verification}

As in [11], we have a large number of atoms $\left({ }^{87} R b\right)$ in a magnetic trap. The atoms are in an hyperfine ground state $\left(\mid F=1, m_{F}=-1>\right)$. A beam of microwave light tuned to the transition $\left|F=1, m_{F}=-1>\rightarrow\right| F=2, m_{F}=$ $0>$ is focused on the trap. The absorption of the microwave photons spinflips atoms into the final state, which do not longer experience the trapping potential and can escape. The released particles fall by the effect of gravity and, downwards they meet atom detectors. These detectors can be of the type considered in [12] (their relevant properties for our experiment will be discussed later). Counting the number of atoms arriving to the detectors we can infer the number of absorptions that have taken place in the trap. This way we have a simple method to measure absorptions. With a very large attenuation of the intensity of the light beam we can expect very few absorptions for each microwave pulse.

A second fundamental element for the scheme is the control of the degree of overlapping in the trap. As signaled before, the effects discussed in this paper depend on the overlapping between the identical particles. As it is well-known in Bose-Einstein condensation or ultracold gases theory the degree of overlapping is related to the thermal wavelength, $\lambda_{T}=h /(2 \pi m k T)^{1 / 2}$ with $T$ the temperature and $k$ Boltzmann's constant. Lowering the temperature of the ensemble of atoms we can make $\lambda_{T}$, directly related to de Broglie's wavelength, comparable to the mean distance between atoms. In this case we hope the proposed effect to manifest. In contrast, for higher temperatures, the overlapping will be in general small and the particles will behave as in product states.

Thus, comparing the number of absorptions in the trap for different temperatures we should observe the modifications of the absorption probabilities as a function of temperature. This verification would correspond to a multi-atom system, not to a two-atom one as discussed in this paper. In consequence, the simple mathematical description given here should be completed taking into account multi-particle symmetrization, or the possibility of collective effects such 
as those discussed in [4]. Depending on the results of that extended analysis, the proposed scheme could be a verification of the dependence of the absorption probabilities on the overlapping, as this depends on the temperature.

\subsection{The quantitative verification}

An extension of the above scheme could deal, in principle, with the two-atom system discussed above. We present separately the different steps of the scheme (see Fig.1).

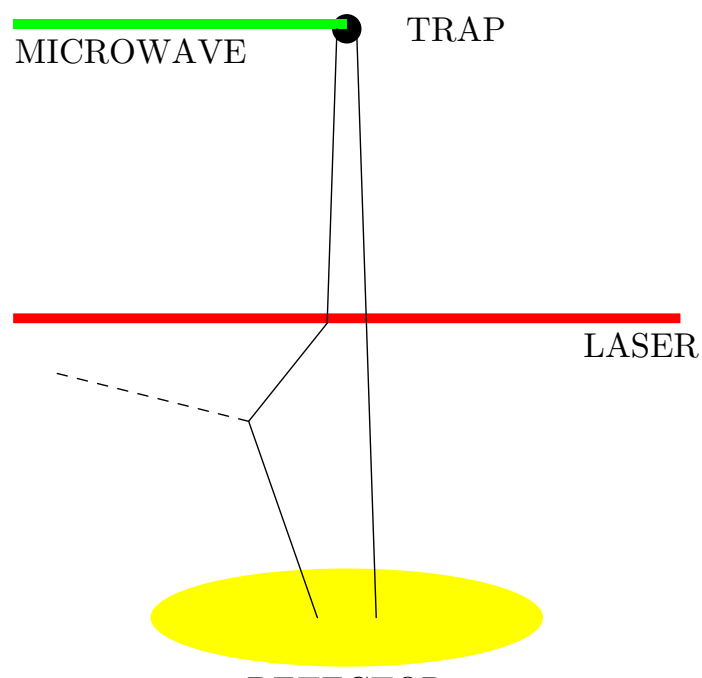

DETECTOR

Figure 1: The continuous lines leaving the trap represent two released atoms. One of them absorbs in the interaction with the laser, emitting spontaneously in the subsequent evolution a photon (discontinuous line). The number of emissions can be counted collecting the photons with lenses and driving them to light detectors.

Preparation of the initial state. As before, a microwave beam releases particles from the trap. The intensity of the beam is attenuated in such a way that only very few atoms (ideally only one) escape with each pulse. In each repetition of the experiment two pulses with a very short tunable delay between them are directed towards the trap. Modulating the temporal delay, we can control the overlapping.

With a postselection process that we shall discuss below we can restrict our considerations to the case of having two released atoms in each run of the experiment. 
Interaction with the laser. Later, the atoms interact with another laser (see Fig. 1). The frequency of this laser is tuned to a transition (not the hyperfine one considered before, which now only has the role of releasing atoms from the trap) of the atom. The intensity of the laser is low enough to only have large probabilities of one-photon absorption.

Note that being the two atoms released at slightly different times, their states at the time of interaction with the laser will be slightly different, depending the overlapping of the delay between the microwave beams.

Spontaneous emission. After the interaction, the atoms continue their downwards propagation. We choose the distance between the interaction region and the detectors long enough for most of the excited atoms return to the ground state by spontaneous emission before reaching the atom detectors. This condition can be easily evaluated using the lifetime of the excited state and the mean velocity of the atoms. By placing photon detectors we can determine the number of spontaneous emission events. In the final step of our scheme the atoms reach the atom detectors. At this stage we must carry out the postselection process to single out the cases with two atoms escaping from the trap. As signaled before we use atom detectors as in [12]. They allow for positionand time-resolved detection events. The second aspect is fundamental because, introducing a temporal window, we can determine if the events have been generated by the two microwave pulses of a single run. The number of spontaneous emissions gives us the number of absorptions in the laser-released atoms interaction. Next, we must compare these numbers for different overlappings between the released atoms. As discussed before, the overlapping can be controlled via the temporal delay between the two microwave pulses. A comparison of the number of absorptions for different delays could test the effects predicted in this paper. Note, as discussed at the end of Sect. 3, that in the case of very similar initial wave functions (very small delays) the changes of the absorption rate are very small and probably undetectable from the experimental point of view. It is for this reason that our proposal to observe the effect focus on the dependence of the absorption rate on the overlapping degree, rather than on the determination of the rate for particular values of the overlapping.

\section{Discussion}

We have shown how the absorption properties of a pair of identical atoms are related to the (anti)symmetric properties of its state. This is another example of the dependence of optical properties of quantum systems on their quantum states.

The process resembles in some aspects the superradiance phenomenon, but we must also emphasize the differences. In superradiance the collective state leads to strong correlations between the atomic dipoles, which are in the basis of the emission enhancement. The collective states originally introduced by 
Dicke had a high degree of symmetry: the atom-radiation description was invariant under atom permutations. This closely resembles our approach, where the (anti)symmetry of the states of the identical atoms generates the modifications of the absorption properties. However, in the case of superradiance, it was later realized that a more realistic description of the process must introduce interactions that break to some extent the permutational invariance [3]. Another difference is that for collective states the properties are independent of the identity (bosonic or fermionic) of the atoms, being only a function of the separation between atoms,....

We also must compare our approach with that associated with entangled states $[2,5,6,7]$. In this case the two mathematical formalisms are similar due to the resemblances of the states describing both systems. As a matter of fact, the (anti)symmetric states are formally identical to entangled states. However, the physical mechanisms underlying both types of non-factorizable states are essentially different. In our case we need proximity between both particles (the overlapping cannot be negligible), whereas the effects associated with entanglement can manifest at large distances. In addition, we can have entangled states giving super- or sub-radiance [2] independently of the atoms identity.

We have proposed an arrangement that could test the existence of the effects discussed in this paper. The simpler version of the arrangement is a simple modification of techniques used in other experiments. It has the inconvenience of only giving a qualitative verification of the effects. The more elaborated version, where the dependence on the initial overlapping could be analyzed experimentally, seems to be more difficult to implement.

Acknowledgments I acknowledge support from Spanish Ministerio de Ciencia e Innovación through the research project FIS2009-09522.

\section{References}

[1] R. H. Dicke, Phys. Rev. 93 (1954) 99.

[2] Z. Ficek, R. Tanaś, Phys. Rep. 372 (2002) 369.

[3] M. Gross, S. Haroche, Phys. Rep. 93 (1982) 301.

[4] J. M. Raimond, P. Goy, M. Gross, C. Fabre, S. Haroche, Phys. Rev. Lett. 49 (1982) 117.

[5] P. Sancho, L. Plaja, J. Phys. B 42 (2009) 165008.

[6] T. Tanabe, T. Odagiri, M. Nakano, Y. Kumagai, I. H. Suzuki, M. Kitajima, N. Kouchi, Phys. Rev. A 82 (2010) 040101(R).

[7] P. Sancho, L. Plaja, Phys. Rev. A 83 (2011) 066101. 
[8] G. S. Agarwal, Phys. Rev. A 83 (2011) 023802.

[9] H. You, S. M. Hendrickson, J. D. Franson Phys. Rev. A 80 (2009) 043823.

[10] R. Loudon, The Quantum Theory of Light, third ed., Oxford University Press, Oxford, 2000.

[11] A. Öttl, S. Ritter, M. Köhl, T. Esslinger, Phys. Rev. Lett. 95 (2005) 090404.

[12] T. Jeltes, J. M. McNamara, W. Hogervorst, W. Vassen, V. Krachmalnicoff, M. Schellekens, A. Perrin, H. Chang, D. Boiron, A. Aspect, C. I. Westbrook, Nature 445 (2007) 402. 